PROCEEDINGS OF THE

AMERICAN MATHEMATICAL SOCIETY

Volume 46, Number 3, December 1974

\title{
TRIPLE POINTS AND SURGERY OF IMMERSED SURFACES
}

\section{THOMAS F. BANCHOFF}

ABSTRACT. For a sufficiently general immersion of a smooth or polyhedral closed 2-dimensional surface into Euclidean 3-space, the number of triple points is congruent modulo 2 to the Euler characteristic. The approach of this paper involves elementary notions of modification of surfaces by surgery.

Let $f: M^{2} \rightarrow E^{3}$ be an immersion in general position of a closed surface $M^{2}$ into Euclidean 3-space so that there are finitely many $N(f)$ triple points of $f$. The purpose of this note is to give a direct and elementary proof of the fact that $N(f) \equiv \chi(M)(\bmod 2)$. The techniques used in this paper are related to the notion of surgery for surfaces, by which a pair of discs is replaced by a cylinder having the same boundary.

The approach used in this paper was reported on at the American Mathematical Society Annual Meeting in Las Vegas, January 1972. With the added assumption of differentiability, it is possible to give a new proof of this same result using normal characteristic classes and singularities of projections, as in [1]. For an immersion $f: M^{2} \rightarrow E^{3}$, set $G_{r}(f)=\left\{x\right.$ in $E^{3} \mid f^{-1}(x)$ consists of precisely $r$ points of $\left.M^{2}\right\}$.

The condition that $f$ is in general position implies that if $x \in G_{r}(f)$ and $f^{-1}(x)=\left\{p_{1}, p_{2}, \cdots, p_{r}\right\}$, then there are disjoint disc neighborhoods $D\left(p_{1}\right), D\left(p_{2}\right), \cdots, D\left(p_{r}\right)$ of these points in $M$ and a homeomorphism $w: B \rightarrow D^{3}$ of a ball neighborhood $B$ of $x$ to the unit ball in $E^{3}$ so that $(w \circ f)\left(D\left(p_{i}\right)\right)$ is the intersection of $D^{3}$ with the plane orthogonal to the $i$ th coordinate vector. If the immersion $f$ is from a particular category, for example, differentiable or piecewise-linear, then we may assume that the homeomorphism $w$ is in the same category, and in fact all the constructions which will be described can be altered in fairly standard ways so

Presented to the Society January 18, 1972; received by the editors September 10 , 1973.

AMS(MOS) subject classfications (1970). Primary 57C35, 57D40, 57D65; Secondary 55A20.

Key words and phrases. Triple points, surgery, immersions, Euler characteristic. 
that the modified immersions remain in the same category.

If $g:[0,1] \rightarrow E^{3}$ is an embedding of the unit interval such that $g .(0,1)$ lies in $G_{r}(f)$ and such that $g(0)$ and $g(1)$ are distinct points of $G_{r+1}(f)$, then we want to describe what we define as a modification of type $r$ along g. We shall assume the following construction lemma:

Given such a map $g$, we may find an embedding $h: Q \rightarrow E^{3}$ of a closed tube $Q=[0,1] \times D^{2}$ into $E^{3}$ such that $h(t, 0)=g(t)$ for each $t$ in $[0,1]$, such that $h\left(\{0\} \times D^{2}\right)=f\left(D\left(p_{r}\right)\right)$ and $h\left(\{1\} \times D^{2}\right)=f\left(D\left(q_{r}\right)\right)$ where $f\left(p_{r}\right)=$ $g(0)$ and $f\left(q_{r}\right)=g(1)$, and such that for each $t$, the set $\{p$ in $M \mid f(p) \epsilon$ $\left.h\left(\{t\} \times D^{2}\right)\right\}$ consists of $2 r$ segments with one endpoint at $h(t, 0)$ and the other at points $a_{0}(t), a_{1}(t), \ldots, a_{2 r-1}(t)$ on the circle $h\left(\{t\} \times \partial D^{2}\right)$. For $0 \leq t \leq 1$, the points $a_{j}(t)$ trace out $2 r$ curves on the image $h(T)$ of the cylinder $T=[0,1] \times \partial D^{2}$.

The modification of type $r$ along $g$ is accomplished by considering the union of $M-\left(D\left(p_{r}\right) \cup D\left(q_{r}\right)\right)$ and the tube $T$ along their boundaries to obtain a new manifold (without boundary) $M^{\prime}$. We define a mapping $f^{\prime}: M^{\prime} \rightarrow$ $E^{3}$ by setting $f^{\prime}(p)=f(p)$ if $p \in M-\left(D\left(p_{r}\right) \cup D\left(q_{r}\right)\right)$ and $f^{\prime}(p)=h(p)$ if $p \in T$. This new mapping $f^{\prime}$ is also an immersion in general position, and we have altered the set $G_{r+1}(f)$ by removing the part lying in $f\left(D\left(p_{r}\right)\right)$ and $f\left(D\left(q_{r}\right)\right)$ and adding the part lying in $G_{r}(t) \cap h(T)$.

Modifications of types 0,1 , and 2 are illustrated in the accompanying figures 0,1 , and 2 .
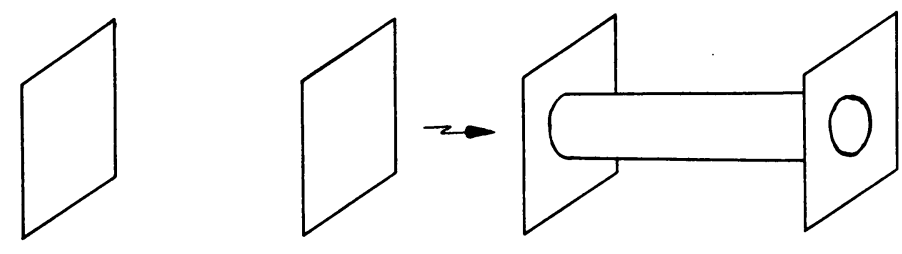

Type 0

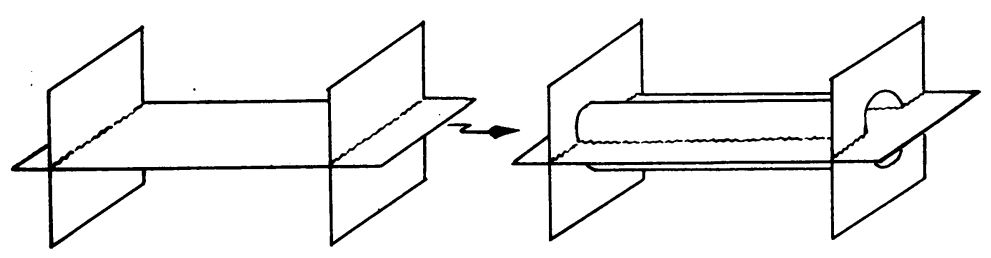

Type 1 


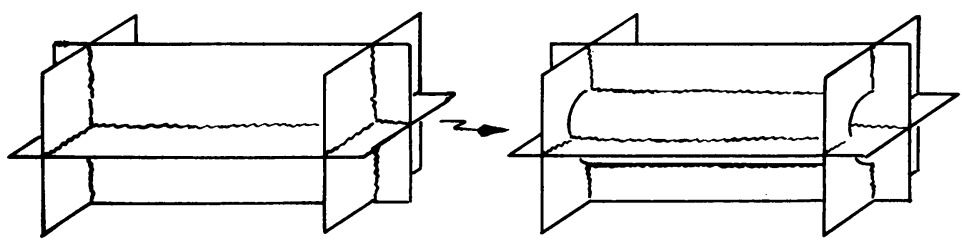

Type 2

By a modification of type 0 we may connect two components of $M$ whose images can be joined by a curve $g:[0,1] \rightarrow E^{3}$ with $g(0,1) \cap f(M)=$ $\varnothing$. We may thus assume that $M$ is connected.

By modifications of type 3 we may remove all or all but one of the triple points on each component of $G_{2}(f) \cup G_{3}(f)$. Then we can use type 2 modifications to connect components of $G_{2}(f)$ and repeat the previous step so that we end up with an immersed surface with either no triple points or with precisely one triple point.

The significant thing about these modifications is that they decrease $\chi(M)$ by 2 and they either leave $N(f)$ the same (for types 0 and 1 ) or decrease $N(f)$ by $2($ for type 3$)$. In any case $\chi\left(M^{\prime}\right)-N\left(f^{\prime}\right) \equiv \chi(M)-N(f)(\bmod 2)$.

Example A. We may construct an immersion $\tilde{f}$ of a surface $\tilde{M}$ with $N(\tilde{f})=1$ as follows. We take the union of three closed unit squares in $E^{3}$ and four triangles together with their edges but not their vertices. We then connect the immersed figure eights on the boundary of this complex by immersed tubes. We obtain in this way an immersion $\tilde{f}$ of a surface $\tilde{M}$ with $\chi(\tilde{M})=3-8=-5$. Thus $\chi(\tilde{M})-N(\tilde{f}) \equiv 0$ (modulo 2 ).

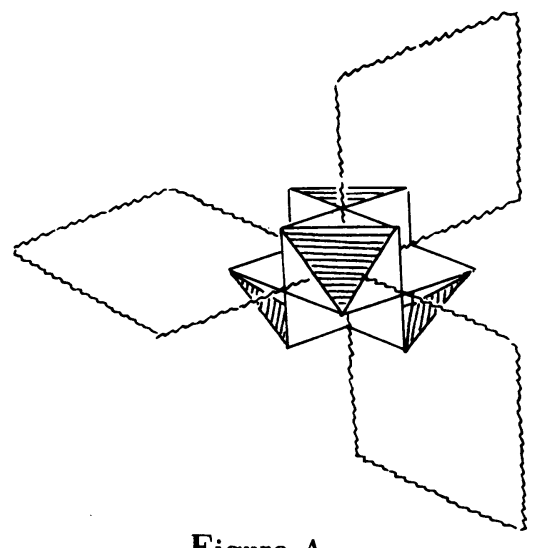

Figure A

Remark. The clebrated immersion of the real projective plane known as Boy's surface is another example of an immersion with odd Euler characteristic $(\chi(P)=1)$ and $N(f)=1$, and that immersion can be obtained by surgery from the one given above. 
We break the proof of the theorem into two propositions which will show that $N(f)$ is even if and only if $\chi(M)$ is even.

Proposition 1. If $N(f)$ is even, then $\chi(M)$ is even.

Proof. By the previous remarks it suffices to prove that $\chi(M)^{\bullet}$ is even if $N(f)=0$. In this case, $G_{2}(f)$ consists of a finite number of disjoint simple closed curves, and on each one we apply a version of the construction lemma as follows.

Let $g: S^{1} \rightarrow E^{3}$ be an embedding of the circle into $G_{r}(f)$ parametrized by $0 \leq t \leq 1$. We then find an embedding $h: Q \rightarrow E^{3}$, where $Q=S^{1} \times D^{2}$ is the solid torus and $h(t, 0)=g(t)$ for all $0 \leq t \leq 1$, such that for each $t$, the set $\left\{p \in M \mid f(p) \in h\left(\{t\} \times D^{2}\right)\right\}$ consists of $2 r$ segments with one endpoint at $h(t, 0)$ and the others at points $a_{0}(t), a_{1}(t), \ldots, a_{2 r-1}(t)$ on the circle $h\left(\{t\} \times \partial D^{2}\right)$. These points trace out $2 r$ curves on the torus $h\left(s^{1} \times \partial D^{2}\right)$, and $a_{0}(1)=a_{i}(0)$ for some index $i$, so $a_{k}(1)=a_{k+i}(0)$ for each index $k$, where the indices are to be reduced modulo $2 r$.

Example 1A. If $r=1$, then we have a curve $g\left(S^{1}\right)$ on $f(M)$ which does not meet $G_{2}(f)$. If $a_{0}(1)=a_{0}(0)$, then $a_{1}(1)=a_{1}(0)$ and $h(Q) \cap f(M)$ is a cylinder which is an orientable neighborhood of the curve $g\left(S^{1}\right)$.

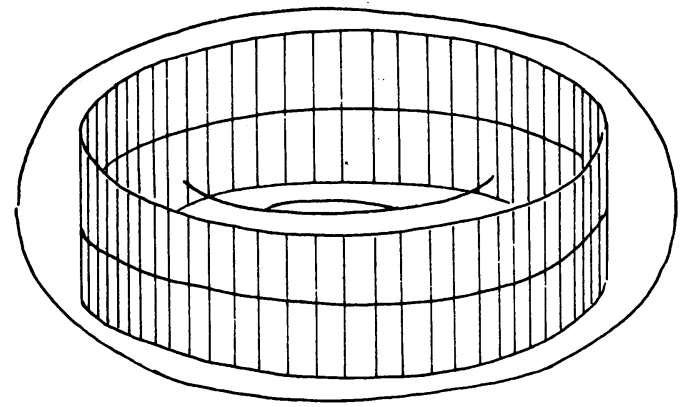

Figure 1A

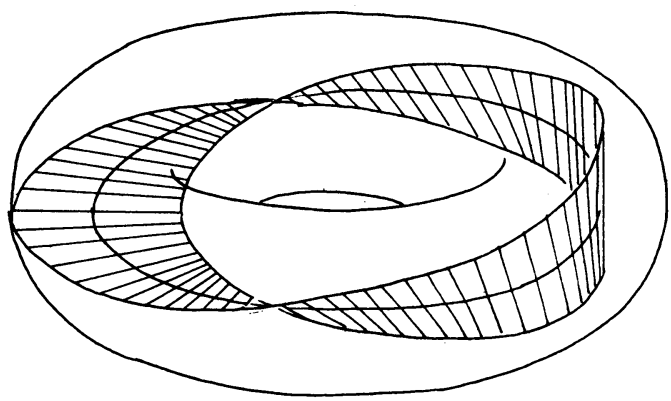

Figure 1B 
Example 1B. If $a_{0}(1)=a_{1}(0)$, so that $a_{1}(1)=a_{0}(0)$, then $h(Q) \cap f(M)$ is a Möbius band which is a nonorientable neighborhood of $g\left(S^{1}\right)$, and $h(T) \cap f(M)$ is a single closed curve which does not disconnect $h(T)$. But in this case we could find a curve on $h(T)$ meeting $h(T) \cap f(M)$ (and $f(M)$ ) at just one point. But this is impossible by a key lemma which is well known in the case of an embedding but which is true also for immersions in general position.

Lemma. If $C$ is a curve which meets $f(M)$ at finitely many points, then there are an even number of points $p$ where $f(p)$ lies in $C$ and $C$ meets the image $f(D(p))$ of $p$ transversally. ( $C$ meets $f(D(p)$ ) transversally if there is a homeomorphism $w: B \rightarrow E^{3}$ of a ball neighborhood of $f(p)$ to the unit ball $D^{3}$ in $E^{3}$ such that $(w \circ f)(D(p))$ is the intersection of $D^{3}$ with the plane orthogonal to the first coordinate axis and such that $w(C) \cap D^{3}$ is the intersection of $D^{3}$ and the first coordinate axis.)

Proof of Lemma. The technique is a standard deformation argumentwe find a one-parameter family of embedded curves $C(u), 0 \leq u \leq 1$, so that $C(0)=C, C(1)$ lies in a neighborhood $B$ disjoint from $f(M)$, and, for all but a finite number of values of $u, C(u)$ meets $f(M)$ transversally at all intersection points. We can assume further that the deformation is itself in general position so that at an exceptional value $\bar{u}$, there is only one point $p$ such that $f(p)$ lies in $C(\bar{u})$ but the intersection is not transversal, and that as $C(u)$ passes through this nontransversal intersection, we either gain two transversal intersections or lose two. Since $C(1)$ does not meet $f(M)$, the curve $C(0)=C$ must meet $f(M)$ transversally at an even number of points.

Corollary. If $S$ is a curve embedded in $M$ which has a nonorientable neighborhood, then $f(S)$ must meet $G_{2}(f)$.

Proof of Corollary. As in the example preceding the Lemma, if $f(S)$ were contained in $G_{1}(f)$, then there would be a curve on the boundary of a tube neighborhood of $f(S)$ meeting $f(M)$ transversally at one point, contradicting the Lemma.

Example 2A. If $r=2$ and if $a_{0}(1)=a_{0}(0)$, then $a_{i}(1)=a_{i}(0)$ for $i=0$, $1,2,3$, and $h(Q) \cap f(M)$ consists of two cylinders intersecting along $g\left(S^{1}\right)$. The four curves in $h(T) \cap f(M)$ separate $h(T)$ into two manifolds-with-' boundary $R_{1}$ and $R_{2}$, each with boundary $h(T) \cap f(M)$, and each consisting of two cylinders.

A modification of type A along $g\left(S^{1}\right)$ is defined by removing the cy- 
linders $h(Q) \cap f(M)$ and attaching the pair of cylinders $R_{1}$ along $h(T) \cap$ $f(M)$. This type of modification removes a component of $G_{2}(f)$ without changing the Euler characteristic.

Example 2B. If $r=2$ and $a_{0}(1)=a_{2}(0)$, then $a_{i}(1)=a_{i+2}(0)$ for $i=$ $0,1,2,3$, and $h(Q) \cap f(M)$ consists of two Möbius bands intersecting along $g\left(S^{1}\right)$. The intersection $h(T) \cap f(M)$ consists of two curves separating $h(T)$ into two cylinders $R_{1}$ and $R_{2}$ each with boundary $h(T) \cap f(M)$.

A modification of type $B$ along $g\left(S^{1}\right)$ is defined by removing the Möbius bands $h(Q) \cap f(M)$ and attaching the cylinder $R_{1}$ along $h(T) \cap f(M)$. Again this type of modification removes a component of $G_{2}(f)$ without changing the Euler characteristic.

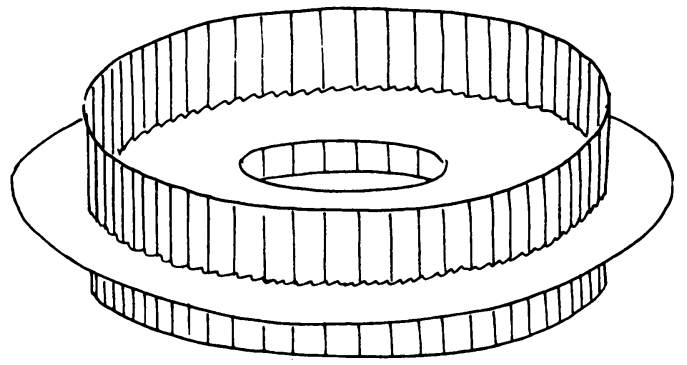

Figure 2A

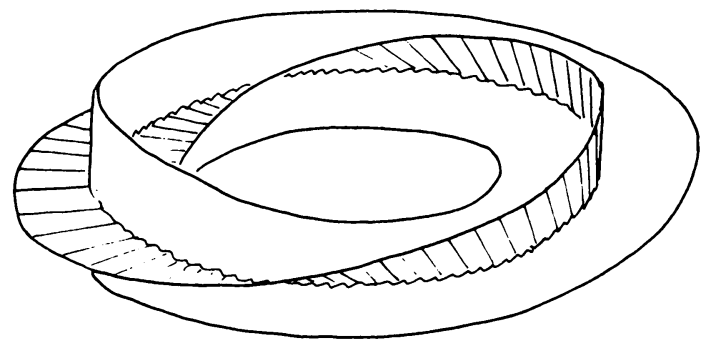

Figure 2B

Example 2C. If $a_{0}(1)=a_{1}(0)$ or $a_{0}(1)=a_{3}(0)$, then the four pieces fit together to form a single curve $h(T) \cap f(M)$ which does not disconnect $h(T)$. But this is impossible for the same reasons that Example 1B cannot occur in an embedded surface.

It follows that if we have an immersion $f: M \rightarrow E^{3}$ in general position with $G_{3}(f)=\varnothing$, then by repeated modification of types $A$ and $B$, we can eliminate all components of $G_{2}(f)$ without changing the Euler characteristic. Note that we might change the number of components of $M$ by modifications of type $A$, and me might change a nonorientable surface into an ori- 
entable manifold by modifications of type $B$. In fact once we have removed all components of $G_{2}(f)$, we are left with an embedded surface which therefore must be orientable. It follows that if $N(f)=0$, then $\chi(M)$ is even, since all orientable surfaces have even Euler characteristic.

Proposition 2. If $N(f)$ is odd, then $\chi(M)$ is odd.

Proof. By translating if necessary, we may assume that the image $f(M)$ is disjoint from the image $\tilde{f}(\widetilde{M})$ constructed in the above Example $A$. We then use a modification of type 0 to obtain a connected manifold $M^{\prime}=\left(M-D\left(p_{1}\right)\right) \cup$ $\left(\tilde{M}-\left(D\left(q_{1}\right)\right) \cup T\right)$ and $f^{\prime}: M^{\prime} \rightarrow E^{3}$ with $N\left(f^{\prime}\right)=N(f)+N(\tilde{f})=$

$N(f)+1$, an even number since $N(f)$ was assumed to be odd. By Proposition 1 , it follows that $\chi\left(M^{\prime}\right)$ is even, and $\chi\left(M^{\prime}\right)=\chi(M)+\chi(\tilde{M})-2$.

Since $\chi(\tilde{M})$ is odd, it follows that $\chi^{(M)}$ is odd.

\section{BIBLIOGR APHY}

1. T. Banchoff, Triple points and singularities of projections of smoothly immersed surfaces, Proc. Amer. Math. Soc. 46 (1974), 402-406.

DEPARTMENT OF MATHEMATICS, UNIVERSITY OF CALIFORNIA, LOS ANGELES, CALIFORNIA 90024

Current address: Department of Mathematics, Brown University, Providence, Rhode Island 02912 